\title{
Thermal and Photo Alignment Behavior of Polyethylene Imine Having Methoxy Substituent Azobenzene Side Chain Group
}

\author{
Mohammad Kamruzzaman ${ }^{1}$, Sun-nam Kim ${ }^{1}$, Yutaka Kuwahara ${ }^{1}$, Tomonari Ogata ${ }^{2}$, \\ Seiji Kurihara ${ }^{1,3,4^{*}}$ \\ ${ }^{1}$ Department of Applied Chemistry and Biochemistry, Graduate School of Science and Technology, Kumamoto University, \\ Kurokami, Kumamoto, Japan \\ ${ }^{2}$ Innovate Collaboration Organization, Kumamoto University, Kurokami, Kumamoto, Japan \\ ${ }^{3}$ PHOENICS, 3-11-38 Higashimachi, Higashi-ku, Kumamoto, Japan \\ ${ }^{4}$ JST-CREST, 5 Sanbancho, Chiyoda-ku, Tokyo, Japan \\ Email: ${ }^{*}$ kurihara@gpo.kumamoto-u.ac.jp
}

Received July 10, 2013; revised August 15, 2013; accepted September 2, 2013

Copyright (C) 2013 Mohammad Kamruzzaman et al. This is an open access article distributed under the Creative Commons Attribution License, which permits unrestricted use, distribution, and reproduction in any medium, provided the original work is properly cited.

\begin{abstract}
Novel type of polyethylene imine having methoxy $\left(-\mathrm{OCH}_{3}\right)$ substituent azobenzene side chain group through eight methylene spacer group (PEI8M) was successfully synthesized and characterized by the polymer by ${ }^{1} \mathrm{H}$ NMR, differential scanning calorimetry, polarized optical microscopic and X-ray diffraction analysis. Synthesized polymer possessed liquid crystalline (LC) properties. Spin coated PEI8M film showed out-of-plane molecular orientation on annealing and non-polarized visible light irradiation. PEI8M in solid film exhibited photoresponsive properties upon irradiation of UV and visible light. PEI8M film also exhibited reversible molecular orientation from random state to out-of-plane and from out-of-plane to random state on annealing, non-polarized UV and visible light irradiation.
\end{abstract}

Keywords: Azobenzene Polymer; Thermal Alignment Behavior; Liquid Crystalline Properties; Out-of-Plane Ordering

\section{Introduction}

In recent years, azobenzene containing polymers have attracted considerable attention for the possibility of changing the molecular orientation by irradiation with an appropriate wavelength of light, and the potential applications including reversible optical storage, holographic grating and optical switching [1,2]. In addition, the azobenzene derivatives are known to undergo trans/cis photoisomerization upon irradiation. This is not a simple switching process between the two photochromic isomers, because a steady state is strongly dependent on the substitution and the wavelength of the excitation.

In literature so far very few papers are published on spontaneous out-of-plane molecular orientation of polymeric liquid crystals (PLCs) [3-7]. Bobrovsky group reported that polyacrylate copolymer containing 4-ethoxy4'-hexoxyazobenzene and cholesterol groups as side

\footnotetext{
*Corresponding author.
}

chains showed spontaneous out-of-plane molecular orientation on a glass substrate by annealing at $75^{\circ} \mathrm{C}$, whereas only partial out-of-plane molecular orientation was observed for polyacrylate homopolymer [3]. Our group also reported that PEI having nitro substituent azobenzene side chain group showed out-of-plane molecular orientation on a glass substrate by annealing but not upon non-polarized visible light irradiation [8]. In addition, Ujiie group reported that some polyethylene imines (PEIs) having azobenzene groups as side groups show liquid crystalline phases and align perpendicular direction to the substrate spontaneously by heating and following cooling without any alignment process $[9,10]$. So, by photochemically controlling the molecular orientation of PEIs between random state and out-of-plane structures, one can fabricate the optical switching system showing excellent memory stability by coating PEIs on a substrate without any alignment process. In this article, we synthesized polyethylene imine having methoxy sub- 
stituent azobenzene side chain group through eight methylene spacer group (PEI8M) and investigated its photochemical as well as thermal alignment behavior on annealing, UV and non-polarized visible light irradiation. And, we also demonstrated the multiple reorientations of the azobenzene chromophores in PEI8M.

\section{Experimental}

\subsection{Materials}

p-Anisidine was purchased from Sigma-Aldrch and used as received. 1,8-dibromooctane was purchased from Tokyo Chemical Industry and polyethylene imine of low molecular weight $(\mathrm{Mn} \approx 1800)$ and high molecular weight $(\mathrm{Mn} \approx 10,000)$ were purchased from Polysciences Inc. Reagents were used without further purification, unless stated. $N, N$-dimethylformamide (DMF) was purchased from Wako Pure Chemical Industries Ltd and was dried up with molecular sieves prior to use.

\subsection{Synthesis of Azobenzene Monomer and Polymer}

\section{Synthesis of 4-methoxy-4'-hydroxyazobenzene (MAz-OH)}

$p$-Anisidine (0.05 mol, $6.16 \mathrm{~g})$ was dissolved in 3 $\mathrm{mol} / \mathrm{L}$ hydrochloric acid $(50 \mathrm{ml})$. After complete dissolution, the solution was cooled with ice to a temperature below $5^{\circ} \mathrm{C}$. With vigorous stirring, to this cold solution was added slowly a solution of $3.5 \mathrm{~g}(0.05 \mathrm{~mol})$ of sodium nitrite in $10 \mathrm{ml}$ of water. The resulting diazonium solution, kept below $5^{\circ} \mathrm{C}$, was subsequently added dropwise to a cold solution of $4.7 \mathrm{~g}(0.05 \mathrm{~mol})$ of phenol in $25 \mathrm{ml}$ of $10 \%$ aqueous sodium hydroxide. The dark brown suspension was acidified and the precipitate was collected. The crude product was washed with copious amount of water and dried under vacuum.

Yield: $65 \%$ as solid. Melting point: $139^{\circ} \mathrm{C}-140^{\circ} \mathrm{C}$. ${ }^{1} \mathrm{H}-\mathrm{NMR}\left(\mathrm{CDCl}_{3}, \delta\right): 7.88(2 \mathrm{H}, \mathrm{dd}$, aromatic), $7.82(2 \mathrm{H}$, $\mathrm{dd}$, aromatic), $6.98(2 \mathrm{H}, \mathrm{d}$, aromatic), $6.94(2 \mathrm{H}, \mathrm{d}$, aromatic), $5.31(1 \mathrm{H}, \mathrm{s},-\mathrm{OH}), 3.87\left(3 \mathrm{H}, \mathrm{s},-\mathrm{OCH}_{3}\right)$. Anal. Calcd for $\mathrm{C}_{13} \mathrm{H}_{12} \mathrm{~N}_{2} \mathrm{O}_{2}$ (228.25): $\mathrm{C}-68.41 ; \mathrm{H}-5.30$; $\mathrm{N}-12.27$. Found: $\mathrm{C}-68.82 ; \mathrm{H}-6.42 ; \mathrm{N}-12.01$.

Synthesis of 4-(8-bromo-n-octyloxy)-4'-methoxy azobenzene [MAzO8Br]

4-(8-bromo-n-octyloxy)-4'-methoxy azobenzene was synthesized by the following procedure: To a mixture of $1.1 \mathrm{~g}$ (4.4 mmol) of 4-methoxy-4'-hydroxyazobenzene (MAz-OH) and $0.91 \mathrm{~g}(6.6 \mathrm{mmol})$ potassium carbonate dissolved in $150 \mathrm{ml}$ of dried acetone, $6.08 \mathrm{~g}(22.3 \mathrm{mmol})$ of 1,8-dibromooctane was added. After refluxing for $48 \mathrm{~h}$ at $70^{\circ} \mathrm{C}$ the reaction mixture was filtered and evaporated the solvent from filtrate and the product $\mathrm{MAzO} 8 \mathrm{Br}$ was re-crystallized from methanol twice. The reaction scheme is shown in Figure 1.

Yield: $81.4 \%$ as solid. Melting point: $107^{\circ} \mathrm{C}-109^{\circ} \mathrm{C}$. Anal. Calcd for $\mathrm{C}_{21} \mathrm{H}_{27} \mathrm{BrN}_{2} \mathrm{O}_{2}$ (419.36): $\mathrm{C}-60.15 ; \mathrm{H}-$ 6.49; N-6.68. Found: $\mathrm{C}-60.25 ; \mathrm{H}-6.35 ; \mathrm{N}-6.70$.

Synthesis of polyethylene imine having azobenzene side chain group (PEI8M)

Polyethylene imines having methoxy substituent azobenzene side chain group with eight methylene spacer groups was synthesized by using different reaction conditions. The typical procedure is as follows: 4-(8-bromon-octyloxy)-4'-methoxy azobenzene $1.76 \mathrm{~g}$ (4.2 mmol), polyethylene imine $0.18 \mathrm{~g}(4.0 \mathrm{mmol})$, potassium carbonate $1.8 \mathrm{~g}(6.0 \mathrm{mmol})$ and $50 \mathrm{ml}$ dry DMF were added into a round bottom flask equipped with a condenser. With continuous stirring the reaction mixture was refluxed at $100^{\circ} \mathrm{C}$ for $72 \mathrm{~h}$. After the reaction, the mixture was filtered and evaporated the half amount of DMF
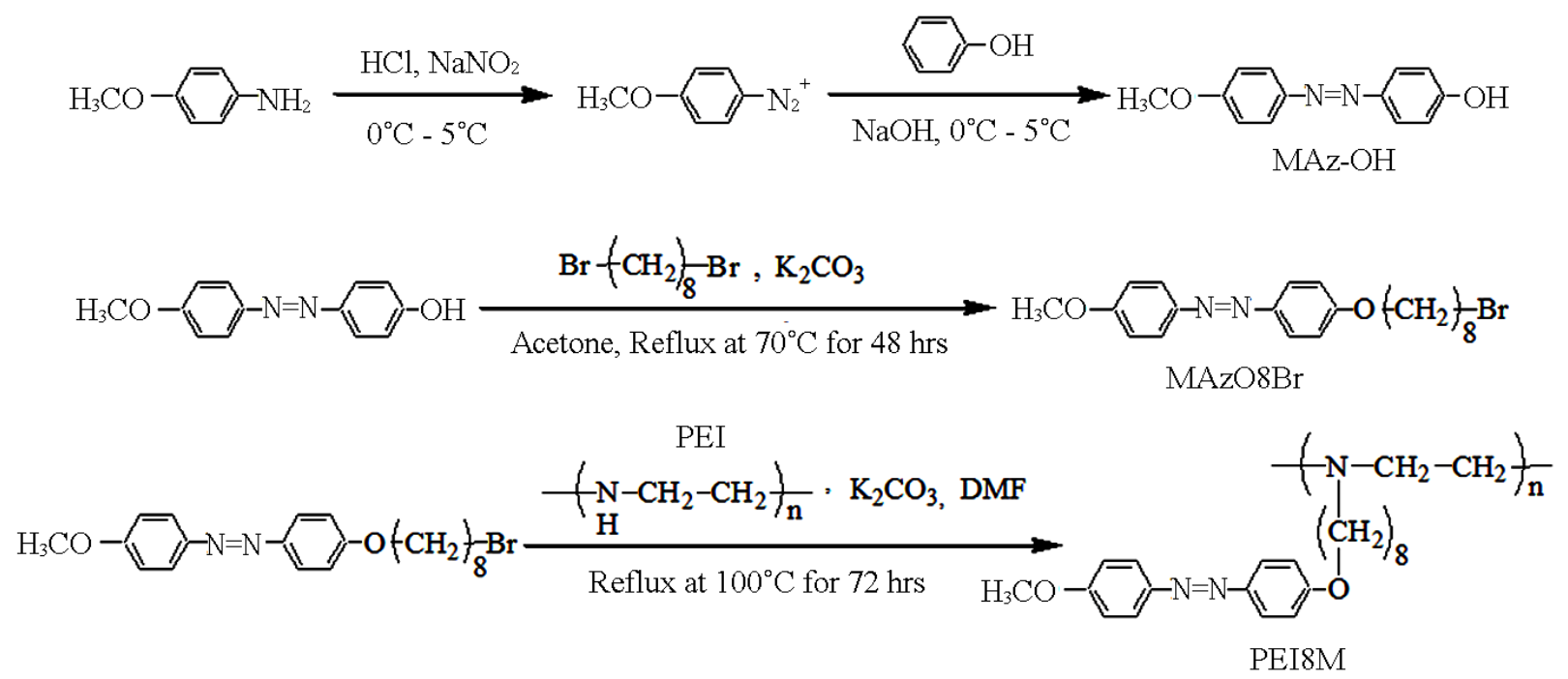

Figure 1. Synthetic route for monomer and polymer. 
from filtrate and then poured into methanol. The resulting polymer (PEI8M) was purified by precipitation from chloroform/THF into methanol two to three times. The removal of the monomer was monitored using thin-layer chromatography. Finally, the product was dried in vacuum for $24 \mathrm{~h}$ (Figure 1).

\subsection{Characterization}

To identify the structure and composition of the synthesized monomers and polymer, elemental analyses were performed with a YANAKO CHN CORDER MT-6. The structures of the compounds were also determined using ${ }^{1} \mathrm{H}$ NMR spectroscopy with $\mathrm{CDCl}_{3}$ or acetone as solvent and tetramethylsilane as internal standard. The spectra were recorded at ambient temperature with a JEOL JNM-EX400, 400MHz NMR-spectrometer.

The phase transition behavior of the polymer was studied by differential scanning calorimetry (DSC; Seiko SSC-5020) with a heating rate of $10 \mathrm{~K} / \mathrm{min}$ and polarizing optical microscopy (Olympus BHSP polarizing microscope; Mettler FP-80 and FP 82 hot stage and controller).

$\mathrm{X}$-ray diffraction was used to confirm the nature of the LC phases and to determine the spacing of the smectic layers. Rigaku, RINT 2100/PC XRD machine (X-Ray, $40 \mathrm{kV} / 200 \mathrm{~mA}$ ) equipped with a $\theta-\theta$ wide angle goniometer and scintillation detector was used for X-ray diffraction (XRD) measurement.

Photoirradiation was performed by using a $500 \mathrm{~W}$ high-pressure $\mathrm{Hg}$ lamp with adequate cut filter for UV and visible light at room temperature. The orientational order was studied using polarized UV-Vis spectroscopy (Perkin Elmer Lambda $650 \mathrm{UV} / \mathrm{V}$ is Spectroscopy) and the angular dependence of the absorbance was measured. The values of order parameter determined by spectro- scopic method were calculated by Equation (1) [3].

$$
S=\frac{A_{\|}-A_{\perp}}{A_{\mid}+2 A_{\perp}}
$$

where $A \|$ is the absorbance at the preferred direction; $A \perp$ is the absorbance perpendicular to this direction.

\section{Results and Discussion}

\subsection{Synthesis and Characterization of PEI8M}

In this study, liquid crystalline polyethylene imine PEI8M was successfully synthesized. For good photo alignment behavior, polyethylene imines should have high degree of substitution. It is assumed that as the introduction of azobenzene group into polyethylene imine main chain increased the out-of-plane alignment behavior of the polymers also increased. So in synthesis step, to achieve higher degree of substitution in PEI, reaction conditions were optimized by changing the reaction parameters such as temperature, reaction time, molar ratio of azobenzene monomer and PEI, molecular weight of PEI and reaction solvent. The degree of substitution of PEI8M has considerable effect on all of these parameters and finally we synthesized PEI8M having 70.2\% degree of substitution and $86.4 \%$ yield by optimizing the reaction conditions as temperature $100^{\circ} \mathrm{C}$, reaction time $72 \mathrm{~h}$, molar ratio of azobenzene monomer to PEI (low mol. wt.) $=4.2: 4.0$, and solvent $(\mathrm{DMF})=50 \mathrm{ml}$. Synthesized polymer showed good solubility in DMF, chloroform, THF and cyclohexanone.

Synthesized polymer, PEI8M was characterized using ${ }^{1} \mathrm{H}$ NMR spectroscopy. The structure and ${ }^{1} \mathrm{H}$ NMR spectrum of the polymer with assignments are shown in Figure 2. The resonances corresponding to the protons of $-\mathrm{OCH}_{3}$ (a) appeared at $3.75 \mathrm{ppm}$, while those of $-\mathrm{CH}_{2} \mathrm{O}-$ (d) at $3.99 \mathrm{ppm}$. The resonances corresponding to phenyl

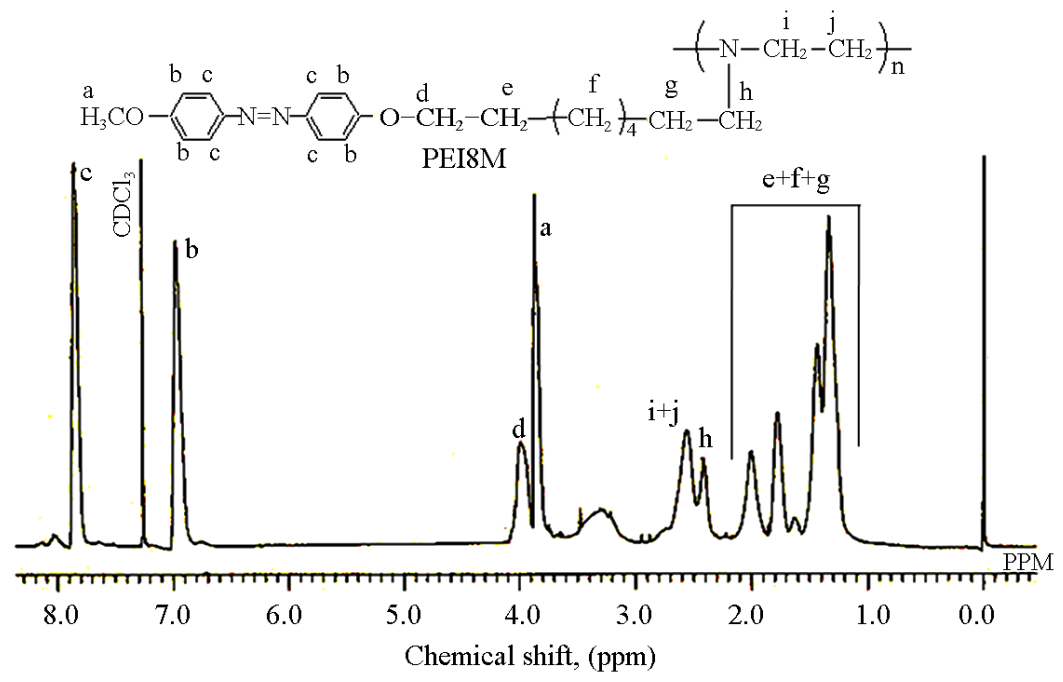

Figure 2. Structure and ${ }^{1} \mathrm{H}$ NMR spectrum of synthesized polyethylene imine PEI8M. 
protons appeared at 6.97 and $7.85 \mathrm{ppm}$ while resonances due to six methylene $\left(-\mathrm{CH}_{2}-\right)(\mathrm{e}, \mathrm{f}, \mathrm{g})$ protons in azobenzene side chain and one methylene $\left(-\mathrm{CH}_{2}-\right)$ (f) protons in polyethylene unit were observed at $1.28-1.92$ $\mathrm{ppm}$. The signal at $2.59 \mathrm{ppm}$ was attributed to methylene protons of $-\mathrm{N}-\mathrm{CH}_{2}-(\mathrm{i}+\mathrm{j})$ and at $2.43 \mathrm{ppm}$ was observed for methylene $\left(-\mathrm{CH}_{2}-\mathrm{N}-\right)$ (h) protons. The chemical shifts and relative intensity of ${ }^{1} \mathrm{H}$ NMR resonances agreed well with the structure shown in Figure 2.

Thermal characteristics of PEI8M were studied using DSC and polarized optical microscopy (POM) analysis. In DSC thermogram, there is a peak at around 116 degree, it is expected for phase transition from nematic to isotropic temperature. In addition, the peak at 98 degree is assessed as phase transition temperature for smectic to nematic. Therefore, the phase transition temperature is determined for G $28 \mathrm{~S} 98 \mathrm{~N} 116 \mathrm{I}$. And, the polymer is stable up to $190^{\circ} \mathrm{C}$.

The synthesized polymer was also characterized by $\mathrm{X}$-ray diffraction measurement. The X-ray diffraction patterns for synthesized polyethylene imine film confirmed that the polymer is smectic layer structures and exhibited strong few diffraction peaks in small angle region. On the basis of X-ray diffraction patterns, the smectic layer spacings were calculated from the XRD peaks using Bragg's law and the results are $2 \theta(1)=2.46 ; 2 \theta(2)$ $=5.06$; dist. $1(\AA)=33.08$ and $\operatorname{dist} .2(\AA)=16.45$.

\subsection{Thermooptical Alignment Behavior of PEI8M in Solid Film}

To study the thermal induced optical anisotropy in PEI8M solid film, the changes in absorbance of spin coated polymeric films on annealing were demonstrated. In this study, all experiments were performed for polymeric films with a thickness ranging from 200 to $300 \mathrm{~nm}$. The films were prepared by spin coating technique. Upon annealing the test film at room temperature, no marked changes took place. However, when the film was kept at the temperature, which is above its glass transition temperature corresponding to LC state, observed the significant spectral changes (Figure 3(a)). On annealing, the absorption in the region of the $\pi-\pi^{*}$ transition is related to out-of-plane ordering was decreased by about $80 \%$. The position of the absorption maximum remained almost unchanged (Figure 3(a)). It should be noted that, the profiles of the corresponding spectra and absorption in the region of the $\varphi-\varphi^{*}$ transitions were preserved.

In addition, the relative intensity of $\pi-\pi^{*}$ absorption band to absorption band around $250 \mathrm{~nm}$ which is assigned to the $\varphi-\varphi^{*}$ transition of the aromatic ring $[4,11]$, was varied as shown in Figure 3(b). It has been reported that the $\varphi-\varphi^{*}$ transition is insensitive to the molecular orientation, consequently, the change in the relative intensity of $\pi-\pi^{*}$ absorption band to $\varphi-\varphi^{*}$ absorption band

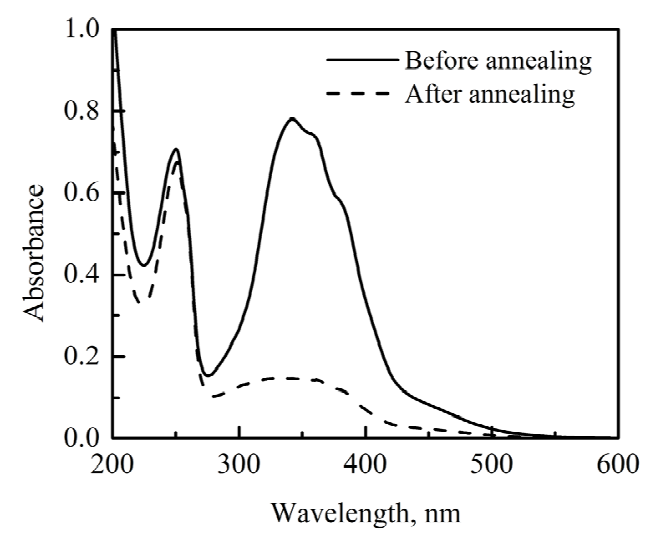

(a)

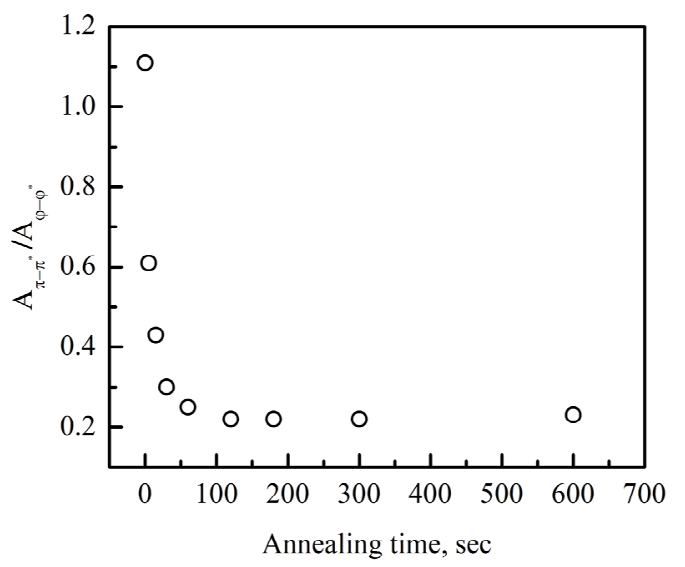

(b)

Figure 3. (a) UV-vis. absorption spectra of PEI8M single layer film before and after annealing at $70^{\circ} \mathrm{C}$ during $5 \mathrm{~min}$. Longer time of annealing does not lead to additional changes. (b) Changes in $\mathbf{A}_{\pi-\pi^{*}} / \mathbf{A}_{\varphi-\varphi^{*}}$ values of PEI8M single layer film on annealing time.

$\left(\mathrm{A}_{\pi-\pi^{*}} / \mathrm{A}_{\varphi-\varphi^{*}}\right)$ is related to the out-of-plane molecular orientation [4,5]. From Figure 3(b), it is also clear that the out-of-plane molecular orientation proceeded in a few minutes.

Therefore, to quantify the thermal induced anisotropy in PEI8M solid film, polarized absorption spectra were measured and explored the angular dependency of absorbance before and after annealing of the film. Annealing of the PEI8M film brought about not only decrease in absorbance corresponding to the $\pi-\pi^{*}$ transition without polarizer, but also change in the polarized absorption spectra as shown in Figure 4. The order parameter (S), the degree of out-of-plane ordering, was calculated from polarized absorption spectra by using Equation (1).

The polarized absorption spectra were recorded at an angle of $45^{\circ}$ to the normal of the film. From polarized absorption spectra, it is clear that, before annealing, little angular dependency was observed, indicating that the azobenzene chromophores were slightly orientated perpendicularly on glass substrate in solid film. But after 


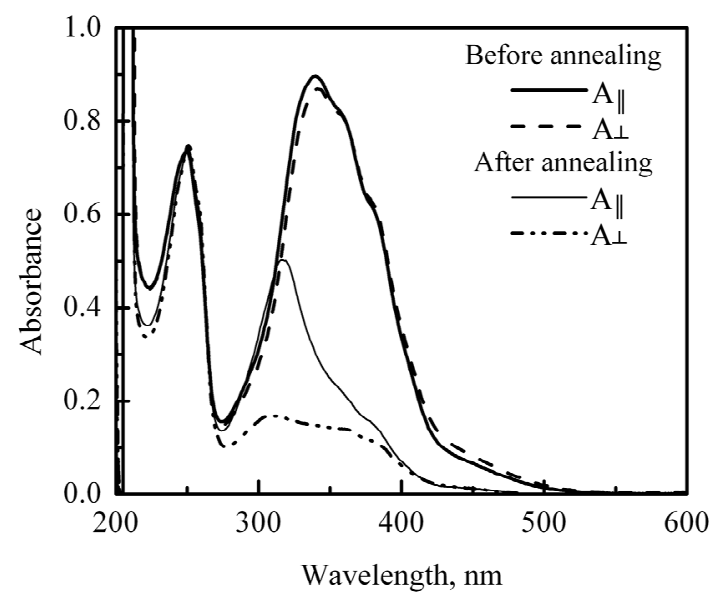

Figure 4. Polarized UV-vis. absorption spectra of PEI8M single layer film before and after annealing at $70^{\circ} \mathrm{C}$ during 5 min. $A_{\|}$and $A_{\perp}$ denote absorption parallel and perpendicular to the polarization direction of actinic light respectively. Spectral measurements were made at $45^{\circ}$ to film normal.

annealing, PEI8M solid film showed big angular dependency and allowing to assess the level of order parameter [3]. The out-of-plane order parameter value before and after annealing were 0.063 and 0.410 respectively.

\subsection{Photo Orientational Behavior of PEI8M in Solid Films}

To investigate the photochemical ordering and photoinduced optical anisotropy in polymeric film, the spectral changes were investigated in polymeric film under irradiation with UV and non-polarized visible light of different wavelengths. Depending on the irradiation wavelength, different steady states of cis-trans photo-isomerization were established allowing or preventing the photo-orientation of azobenzene chromophores in PEI8M.

Upon irradiation of non polarized UV light on spin coated solid films of PEIs, significant spectral changes were observed in polymers which are typical for transcis photoisomerization [12-14]. In PEI8M film, photostationary state with a little high content of the cis isomeric form was achieved within 2 or $3 \mathrm{~min}$ as shown in Figure 5(a). In film, due to molecular aggregation, trans-cis photoisomerization rate is not as high as in solution. On the subsequent irradiation with visible light of $435 \mathrm{~nm}$, the reverse changes occurred establishing a steady state with a high concentration of trans isomers (Figure 5(b)). The recovery of the initial spectrum was complete, even the value of absorption maxima corresponding to $\pi-\pi^{*}$ transition exceeded the initial value. This phenomenon was related to the partial out-of-plane molecular ordering of azobenzene moieties on glass substrate.

Upon a prolonged irradiation ( $>5 \mathrm{~s}$ ), one may observe a decrease in the absorption in both regions corresponding to the $\pi-\pi^{*}$ and $n-\pi^{*}$ transitions as shown in Figure 5(c). Within the first 5 seconds of irradiation with visible light, the back cis-trans photoisomerization occurred, while upon continued irradiation, the azobenzene groups

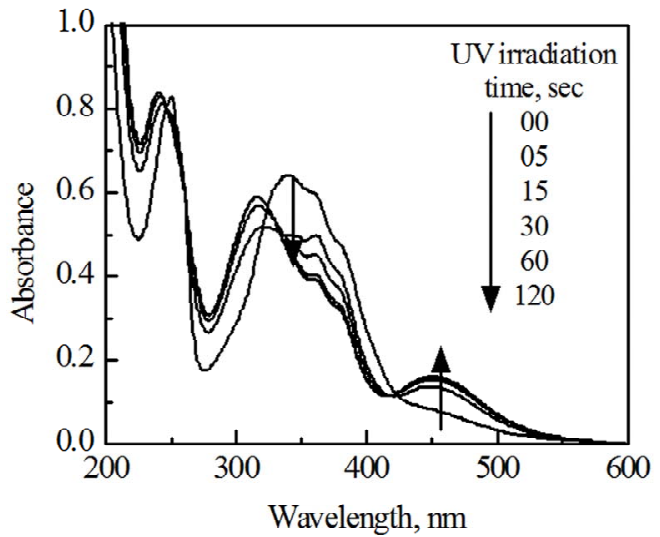

(a)

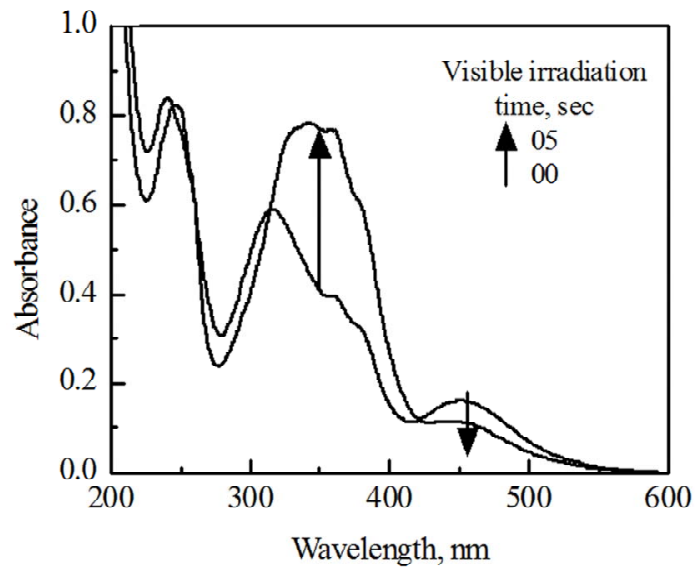

(b)

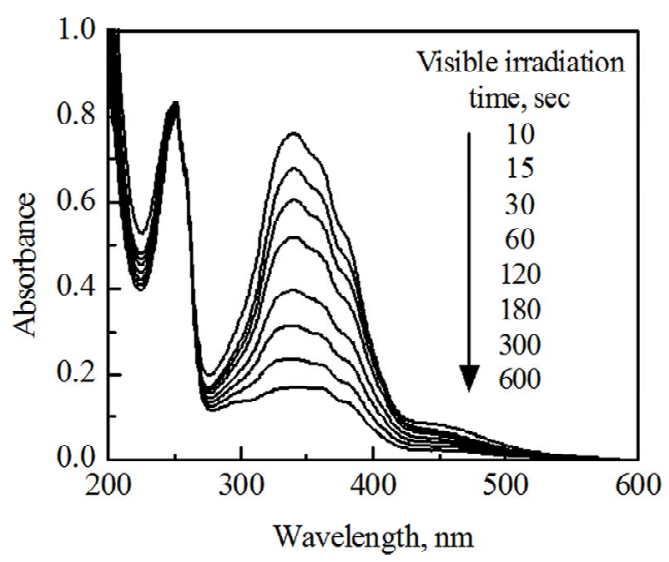

(c)

Figure 5. Changes in UV-vis. absorption spectra of PEI8M polymeric film during (a) UV irradiation $(365 \mathrm{~nm})$, (b) first $05 \mathrm{sec}$ visible light irradiation $(435 \mathrm{~nm})$ and (c) further visible light irradiation $(435 \mathrm{~nm})$. The intensity of $\mathrm{UV}$ and visible light was $7.0 \mathrm{mWcm}^{-2}$ and $80 \mathrm{mWcm}^{-2}$ respectively. 
were oriented along the normal of film due to photoorientation [3]. In this case, the transition moment of these groups become oriented perpendicular to the plane of film; as a result, the probability of light absorption and, thus, optical density markedly decreased [3]. So irradiation with non-polarized visible light $(435 \mathrm{~nm})$ caused the induction of anisotropy in the films by photoorientation of azobenzene side groups.

\subsection{Reversibility of Alignment Ordering of PEI8M in Solid Film}

In order to show the possibility of reversible reorientation of azobenzene chromophores in PEI8M, molecular ordering behaviors were observed at different conditions. And synthesized polymer showed reversible molecular alignment behavior from random state to out-of-plane order and from out-of-plane order to random state. In all cases we observed the angular dependency on absorbance at different conditions and plotted the polar plots. Figure 6 illustrates the polar plots for PEI8M, showing the changes in absorbance on annealing, UV and nonpolarized visible light irradiation. The shape of the corresponding polar plots at various conditions has demonstrated the nature of the alignment behaviors.

The reversibility of the alignment behavior of PEI8M was also demonstrated at different conditions in terms of order parameter, estimated by measuring polarized absorption spectra. In fresh film, polymer showed no out-of-plane ordering $(\mathrm{S}=0.04)$. Upon annealing, polymeric film showed high order parameter value $(S=0.41)$ showing out-of-plane molecular alignment. Irradiation with the $365 \mathrm{~nm}$ light brings the film always into the isotropic state and erases any previously induced anisotropy. So after the irradiation of UV light on annealed films, thevalue of order parameter decreased via cis-trans photoi-

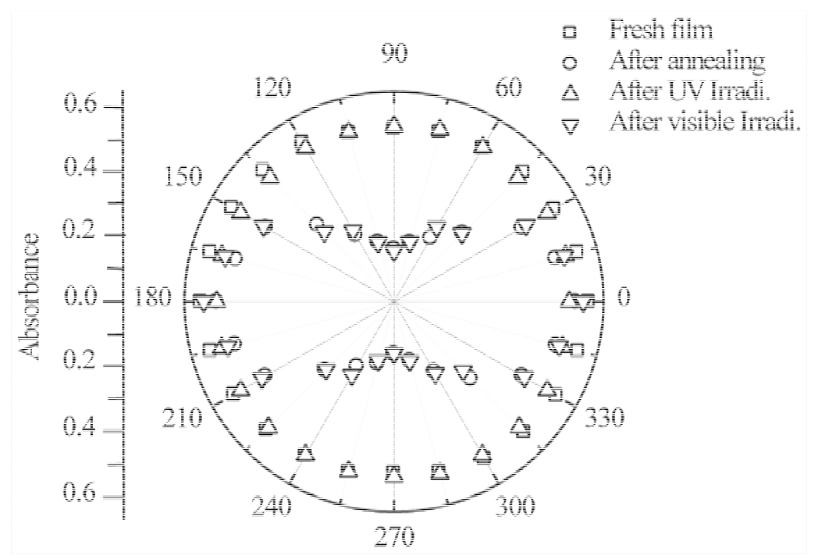

Figure 6. Polar plot for PEI8M $(\lambda=317 \mathrm{~nm})$ single layer film: $\square$-fresh film, $O$-after annealing, $\Delta$-after non-polarized UV light irradiation and $\nabla$-after non-polarized visible light irradiation. Spectral measurements were made at $45^{\circ}$ to film normal. somerization and reached to almost initial level ( $\mathrm{S}=$ 0.024). Namely the azobenzene molecules aligned randomly on the glass substrate. Following non-polarized visible light irradiation again increased the order parameter value from 0.024 to 0.44 , indicating the transformation of molecular orientation of azobenzene molecules from random state to out-of-plane order due to photo-orientation. The efficiency of the induction of anisotropy in the polymeric film was higher for the $435 \mathrm{~nm}$ non-polarized visible light compared with that of annealing because the pre-irradiation with the $365 \mathrm{~nm}$ light strongly increased the effectiveness of photoorientation process [15]. Again upon UV irradiation, the azobenzene group aligned randomly on the glass substrate $(S=0.02)$. It should be noted that after each step of irradiation with the $365 \mathrm{~nm}$ UV light the angular-dependent spectra were exactly the same. This indicates a complete erasure of the previously written orientation was achieved. Again, after annealing the out-of-plane order parameter value increased from 0.02 to 0.41 and after irradiation of $435 \mathrm{~nm}$ non-polarized visible light order parameter value changed from 0.01 to 0.46 . In this way, the reversibility of the alignment behavior of azobenzene groups in polyethylene imine could be achieved by changing the conditions such as annealing, UV and non-polarized visible light irradiation. All these results indicated that the optical information in the polymer can be rewritten in the system without any memory effects of previous irradiation or annealing.

\section{Conclusion}

Liquid crystalline polyethylene imine (PEI8M) having methoxy $\left(-\mathrm{OCH}_{3}\right)$ substituent azobenzene side chain group through eight methylene spacer group was successfully synthesized and characterized. Photochemical, thermooptical as well as photoorientational behavior of the polymer were investigated elaborately. Spin coated PEI8M film showed out-of-plane molecular ordering on annealing and exhibited photoresponsive properties upon irradiation of UV and visible light. PEI8M film also exhibited reversible molecular orientation from random state to out-of-plane and from out-of-plane to random state on annealing, non-polarized UV and visible light irradiation. This reversibility of molecular ordering has been achieved by the combination of thermal and photochemical processes.

\section{REFERENCES}

[1] K. Ichimura, "Photoalignment of Liquid-Crystal Systems," Chemical Review, Vol. 100, No. 5, 2000, pp. 1847-1873. http://dx.doi.org/10.1021/cr980079e

[2] T. Ikeda, "Photomodulation of Liquid Crystal Orientations for Photonic Applications," Journal of Materials 
Chemistry, Vol. 13, No. 9, 2003, pp. 2037-2057. http://dx.doi.org/10.1039/b306216n

[3] A. Bobrovsky, N. Boiko, V. Shibaev and J. Stumpe, "Comparative Study of Photoorientation Phenomena in Photosensitive Azobenzene-Containing Homopolymers and Copolymers," Journal of Photochemistry and Photobiology A: Chemistry, Vol. 163, No. 3, 2004, pp. 347-358. http://dx.doi.org/10.1016/j.jphotochem.2004.01.021

[4] B. Sapich, A. B. E. Vix, J. P. Rabe, J. Stumpe, G. Wilbert and R. Zentel, "Ordering and Dewetting in Spin-Coated Films of a Liquid Crystalline Main Chain Polymer," Thin Solid Films, Vol. 514, No. 1, 2006, pp. 165-173. http://dx.doi.org/10.1016/j.tsf.2006.02.021

[5] T. Uekusa, S. Nagano and T. Seki, "Highly Ordered InPlane Photoalignment Attained by the Brush Architecture of Liquid Crystalline Azobenzene Polymer," Macromolecule, Vol. 42, No. 1, 2009, pp. 312-318.

[6] S. Ujiie and K. Iimura, "Thermal Properties and Orientational Behavior of a Liquid-Crystalline Ion Complex Polymer," Macromolecules, Vol. 25, No. 12, 1992, pp. 31743178. http://dx.doi.org/10.1021/ma00038a024

[7] S. Ujiie and K. Iimura, "Formation of Smectic Orientational Order in an Ionic Thermotropic Liquid-Crystalline Side-Chain Polymer," Polymer Journal, Vol. 25, No. 4, 1993, pp. 347-354. http://dx.doi.org/10.1295/polymj.25.347

[8] M. Kamruzzaman, Y. Kuwahara, T. Ogata, S. Ujiie and S. Kurihara, "Synthesis, Thermal, and Photo Alignment Behavior of Polyethylene Imines Having Nitro Substituent Azobenzene Side Chain Group," Journal of Applied Polymer Science, Vol. 120, No. 2, 2011, pp. 950-959. http://dx.doi.org/10.1002/app.33138
[9] S. Ujiie and K. Iimura, "Ammonium Halide Type Thermotropic Liquid-Crystalline Polyethyleneimines and Those Low-Mass Model Compounds," Chemistry Letters, Vol. 19, No. 6, 1990, pp. 995-998.

http://dx.doi.org/10.1246/cl.1990.995

[10] S. Ujiie and Y. Yano, "Thermotropic and Lyotropic Behavior of Novel Amphiphilic Liquid Crystals Having Hydrophilic Poly(ethyleneimine) Units," Chemistry Communications, Vol. 1, 2000, pp. 79-80.

[11] J. Fabian and H. Hartmann, "Light Absorption of Organic Colarants," Springer-Verlag, Berlin, 1980, pp. 32-79. http://dx.doi.org/10.1007/978-3-642-67587-4

[12] A. Natansohn and P. Rochon, "Photoinduced Motions in Azo-Containing Polymers," Chemical Reviews, Vol. 102, No. 11, 2002, pp. 4139-4175. http://dx.doi.org/10.1021/cr970155y

[13] M. Z. Alam, T. Ohmachi, T. Ogata, T. Nonaka and S. Kurihara, "Photoisomerization Behavior and Photoinduced Surface Relief Gratings on Azopolymer Film by a Monochromatic Light Irradiation," Optical Materials, Vol. 29, No. 4, 2006, pp. 365-370. http://dx.doi.org/10.1016/j.optmat.2005.10.005

[14] M. Ishiguro, D. Sato, A. Shishido and T. Ikeda, "BraggType Polarization Gratings Formed in Thick Polymer Films Containing Azobenzene and Tolane Moieties," Langmuir, Vol. 23, No. 1, 2007, pp. 332-338. http://dx.doi.org/10.1021/la061587i

[15] D. Rais, Y. Zakrevskyy, J. Stumpe, S. Nešpůrek and Z. Sedláková, "Photoorientation of Azobenzene Side Groups in a Liquid-Crystalline Polybutadiene-Based Polymer," Optical Materials, Vol. 30, No. 8, 2008, pp. 1335-1342. http://dx.doi.org/10.1016/j.optmat.2007.07.001 